Article

\title{
Non-Rotationally Symmetric Field Mapping for Back-Scanned Step/Stare Imaging System
}

\author{
Qiang Fu ${ }^{1,2, *}$, Xin Zhang ${ }^{1}$, Jianping Zhang ${ }^{1}$, Guangwei Shi ${ }^{1}$, Shangnan Zhao ${ }^{1}$ \\ and Mingxin Liu ${ }^{1}$ \\ 1 Key Laboratory of Optical System Advanced Manufacturing Technology, Changchun Institute of Optics, Fine \\ Mechanics and Physics, Chinese Academy of Sciences, Changchun 130033, China; optlab@ciomp.ac.cn (X.Z.); \\ zhjp-ycy@163.com (J.Z.); sgwzhy@126.com (G.S.); 18810575846@163.com (S.Z.); 13261531101@163.com (M.L.) \\ 2 Daheng College, University of Chinese Academy of Sciences, Beijing 100049, China \\ * Correspondence: fuqianghit@163.com; Tel.: +86-0431-8670-8669
}

Received: 22 February 2020; Accepted: 27 March 2020; Published: 1 April 2020

check for updates

Featured Application: The work in this paper can be used to guide optical system design for step/stare imaging with focal plane arrays (FPAs). For infrared search and track (IRST) and other applications, when utilizing the fast steering mirrors to maintain the line of sight stability, they always face the problem of field points wandering during back-scanning. This paper proposes a non-rotationally symmetric field mapping method for the back-scanned step/stare imaging system, which can make all field points stable on the FPA during back-scanning.

Abstract: Step/stare imaging with focal plane arrays (FPAs) has become the main approach to achieve wide area coverage and high resolution imaging for long range targets. A fast steering mirror (FSM) is usually utilized to provide back-scanned motion to compensate for the image motion. However, the traditional optical design can just hold one field point relatively stable, typically the central or on-axis field point, on the FPA during back-scanning; all other field points may wander during the exposure due to imaging distortion characteristics of the optical system, which reduces the signal to noise ratio (SNR) of the target. Aiming toward this problem, this paper proposes a non-rotationally symmetric field mapping method for the back-scanned step/stare imaging system, which can make all field points stable on the FPA during back-scanning. First of all, the mathematical model of non-rotationally symmetric field mapping between object space and image space is established. Then, a back-scanned step/stare imaging system based on the model is designed, in which this non-rotationally symmetric mapping can be implemented with an afocal telescope including freeform lenses. Freeform lenses can produce an anamorphic aberration to adjust distortion characteristics of the optical system to control image wander on an FPA. Furthermore, the simulations verify the effectiveness of the method.

Keywords: infrared search and track; infrared imaging system; step/stare; fast steering mirror

\section{Introduction}

Modern intelligence, surveillance, and reconnaissance systems strongly require to have wide area coverage and high-resolution imaging abilities. High-resolution imaging has often been achieved at the cost of a small field of view (FOV) for current electro-optical and infrared sensors [1]. In certain imaging applications, such as infrared search and track (IRST) systems, it is desirable for the imaging sensor to scan large fields of regard at a high rate and with diffraction limited performance.

The second-generation IRST systems utilize long line detectors with time delay and integration capability to achieve wide area coverage. However, due to systems limitations of line detectors, 
the scanning mode of IRST cannot be stopped at any desired position to scan a small sector of interest. They also suffer from more false alarms [2].

The third-generation IRST systems utilize the FPAs with step/stare imaging to achieve wide area coverage. The new IRST achieves greater performances than the previous generation thanks to their staring nature, i.e., to longer integration times (milliseconds rather than microseconds) [3-8]. In step/stare imaging applications, a high-resolution narrow FOV sensor is rapidly stepped or indexed across an area of interest, stopping at intervals to stare and collect imagery. Then the images at each location are stitched together to form a large but high-resolution image. This process requires a series of small angular steps and a high angular acceleration [9].However, the image motion occurs during the scanning within the exposure time. In order to achieve the line of sight stability, a special optical mechanism has been required to compensate the scanning image motion to achieve the "stare" result when searching and tracking $[10,11]$.

Among different technical methods to achieve step/stare imaging, the back-scanning optical compensation technology is the most commonly utilized, in which a plane mirror within the optical system moves reversely to achieve the line of sight stability when searching and tracking [12-16]. For example, the camera on the Global Hawk unmanned aerial vehicle utilizes the fast steering mirror (FSM) behind the afocal optics to compensate the scanning image motion. FSMs are utilized to point for line of sight stability in optical systems. With small size, high accuracy, high bandwidth and fast response speed, FSMs are widely applied in electro-optical and infrared sensors [17-19].

However, it is frequently assumed that the virtual of a target viewed through a rotating mirror moves with respect to the observer at twice the angular rate of mirror rotation, which is inaccurate and leads to imprecise treatment of open-loop tracking systems. The reason is that the sample of rays reflected from a target to an arbitrary tracker position changes as a function of mirror orientation, and the angle of incidence is not a linear function of mirror orientation, but depends on the relative positions of the target, mirror, and tracker, as well as the orientation of the mirror [20,21].Although the equations describing the line-of-sight kinematics derive entirely from the simple plane mirror law of reflection, they are non-linearly- and axis-coupled and these effects increase as the FSM angular displacement increases, which would contribute to pointing errors in certain modes of operation [9].

As discussed above, the back-scanned step/stare imaging system can scan a large field of regard at a high rate and with diffraction limited performance. However, the traditional optical design forms can hold only one field point relatively stable on the FPA, typically the central or on-axis field point; all other off-axis field points may wander during the exposure time due to image distortion characteristics of the imaging systems, which reduces the SNR of the target. Even though the imaging systems can be designed for high performance imaging and have no distortion when there is no motion, the image wander and image blur for off-axis field points still exist. The amount and significance of the blurring depends on the angular change of the FSM, the angular FOV, and the number of pixels (on the FPA) across the FOV. This problem becomes apparent with the increase of the angle change of FSMs, the increase of the FOV and the decrease of the pixel pitch. Now staring arrays have been fabricated in the mid-wave infrared (MWIR) on the format order of $8 \times 8 \mathrm{~K}$ with $10 \mu \mathrm{m}$ pitch [22-24]. Long-wave infrared (LWIR) detectors are not as advanced as MWIR detectors, but they are also making significant progress in larger format size and smaller detector pitch [18]. In this situation, the sensors with super definitions FPAs and long focal length require tight control on the imaging distortion, and even a single pixel shift may cause too much blur for acceptable system performance. Step/stare and IRST systems are widely applied, but this problem of image blurring due to off-axis field point wander is not well recognized and solved.

Therefore, the research objective is to find optimal field mapping between object space and image space, which would make all field points stable on the FPA during back-scanning and the image blurring could be mitigated. At the same time, we want to design a proper optical system that can implement these field mappings. In this manuscript, the mathematical model of non-rotationally symmetric field mapping between object space and image space is established in Section 2. A design result of 
back-scanned step/stare imaging system based on this model is designed in Section 3. Experimental simulation results are analyzed in Section 4.

\section{Mathematical Model for Back-Scanned Step/Stare Imaging System}

Figure 1 is a principle schematic diagram of utilizing the plane mirror to achieve the line of sight stability. It is assumed that the optical axis and the mirror are in the state $\mathrm{A}$ at the beginning of the exposure of the FPA. At this time, the light beam from the infinity object point on the optical axis is turned 90 degrees by the plane mirror, and then focused on the center of the FPA by the imager. During the exposure process, with the movement of the platform, the optical axis changes to the state $B$. The angle between the original optical axis $A$ and the current optical axis $B$ is $\alpha$. By turning the mirror to the state $B$, the angle between the mirror $A$ and the mirror $B$ is $\alpha / 2$. The light beam from the infinity object point on the optical axis is turned by the mirror, and then still focused on the center of the FPA by the imager. The change of the optical axis is compensated by the change of the angle of the mirror. During the integration time of the FPA, the light beam from the infinity object point on the optical axis is always focused on the center of the FPA.

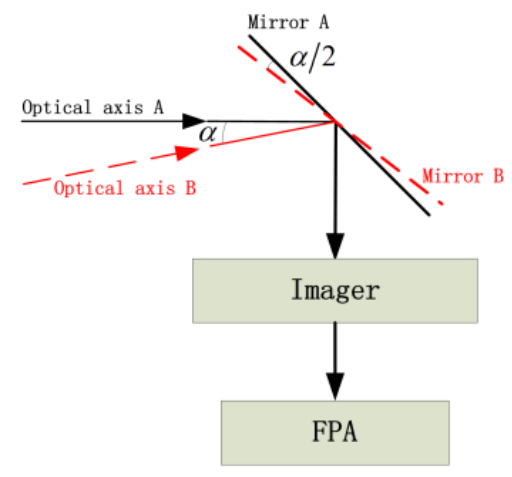

Figure 1. A schematic diagram of utilizing the plane mirror to achieve the line of sight stability.

During the rotation of the mirror, projection distortion is produced in the image plane due to the change of the optical axis. This projection distortion makes off-axis field points wander during the exposure time, which reduces the SNR of the target. In the following, we want to find optimal field mapping between object space and image space, which would make all field points stable on the FPA during back-scanning and the image blurring could be mitigated.

It is assumed that the relationship among the image height, the focal length of the imager and the incident angle of the imaging system in Figure 1 can be expressed by Equation (1):

$$
h=f^{\prime} \tan \theta
$$

where $h$ is the image height, $f^{\prime}$ is the focal length of the imager, $\theta$ is the angle between the incident light and the optical axis.

In Figure 2, the FOVs in object space are conventionally defined for optics, based on the $x-y-z$ coordinate system.

When the optical axis is in the state A, the image point coordinates of the chief ray OI on the FPA are shown in Equation (2),

$$
\left\{\begin{array}{l}
h_{x}=f^{\prime} \tan \left(\theta_{x}\right) \\
h_{y}=f^{\prime} \tan \left(\theta_{y}\right)
\end{array}\right.
$$

where $h_{x}$ is the image height in $\mathrm{x}$ direction, $h_{\mathrm{y}}$ is the image height in $\mathrm{y}$ direction. 
Considering that $\mathrm{y}$-direction of the FOV is the scanning direction. When the change angle of the optical axis is $\alpha$ and the mirror rotates $\alpha / 2$, the image point coordinates $\left(h_{x^{\prime}}{ }^{\prime} h_{y}{ }^{\prime}\right)$ of the chief ray OI on the FPA will come to be expressed by Equation (3):

$$
\left\{\begin{array}{l}
h_{x}{ }^{\prime}=f^{\prime} \tan \left(\theta_{x}\right) \frac{\cos (\alpha)\left[1+\tan ^{2}(\alpha)\right]}{1+\tan \left(\theta_{y}\right) \tan (\alpha)} \\
h_{y}{ }^{\prime}=f^{\prime}\left[\tan \left(\theta_{y}-\alpha\right)+\tan (\alpha)\right]
\end{array}\right.
$$

Calculating the difference between image points $\left(h_{x}{ }^{\prime}, h_{y}{ }^{\prime}\right)$ and $\left(h_{x}, h_{y}\right)$ in Equation (4),

$$
\left\{\begin{array}{l}
h_{x}{ }^{\prime}-h_{x}=f^{\prime} \tan \left(\theta_{x}\right)\left[\frac{1}{\cos (\alpha)+\tan \left(\theta_{y}\right) \sin (\alpha)}-1\right] \\
h_{y}{ }^{\prime}-h_{y}=f^{\prime}\left[\tan \left(\theta_{y}-\alpha\right)+\tan (\alpha)-\tan \left(\theta_{y}\right)\right]
\end{array}\right.
$$

it can be seen that the position of the image point of the chief ray OI changes during back-scanning.

It is assumed that the relationship among the image height, the focal length of the imager and the incident angle of the imaging system in Figure 1 is shown in Equation (5):

$$
h=f^{\prime} \theta
$$

When the optical axis is in the state A, the image point coordinates of the chief ray OI on the FPA are expressed by Equation (6):

$$
\left\{\begin{array}{l}
h_{x}=f^{\prime} \theta_{x} \\
h_{y}=f^{\prime} \theta_{y}
\end{array}\right.
$$

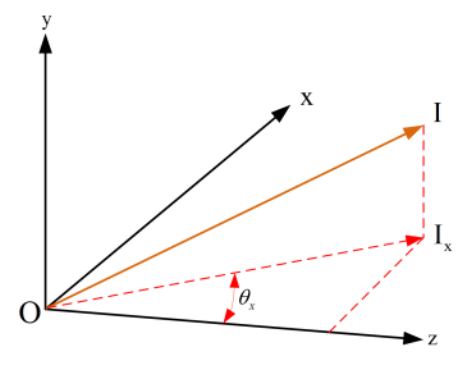

(a)

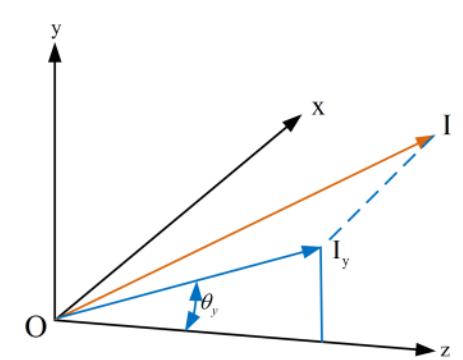

(b)

Figure 2. The field of views (FOVs) in object space are conventionally defined for optics, based on the $x-y-z$ coordinate system. The optical axis is Oz. OI is the chief ray of a certain FOV. (a) The projection of OI on the $\mathrm{xOz}$ plane is $\mathrm{OI}_{\mathrm{x}}$. The angle between $\mathrm{OI}_{\mathrm{x}}$ and $\mathrm{Oz}$ is $\theta_{x}$, which is defined as the FOV in $\mathrm{x}$ direction of the chief ray OI. (b) The projection of OI on the $y O z$ plane is $\mathrm{OI}_{\mathrm{y}}$. The angle between $\mathrm{OI}_{\mathrm{y}}$ and $\mathrm{Oz}$ is $\theta_{y}$, which is defined as the FOV in y direction of the chief ray OI.

Considering that y-direction of the FOV is the scanning direction. When the change angle of the optical axis is $\alpha$ and the mirror rotates $\alpha / 2$, the image point coordinates $\left(h_{x^{\prime}}{ }^{\prime} h_{y}{ }^{\prime}\right)$ of the chief ray OI on the FPA will come to be expressed by Equation (7):

$$
\left\{\begin{array}{c}
h_{x}^{\prime}=f^{\prime} \tan ^{-1}\left[\tan \left(\theta_{x}\right) \cos (\alpha)\right] \\
h_{y}^{\prime}=f^{\prime} \theta_{y}
\end{array}\right.
$$


Calculating the difference between image points $\left(h_{x}{ }^{\prime}, h_{y}{ }^{\prime}\right)$ and $\left(h_{x}, h_{y}\right)$ in Equation (8),

$$
\left\{\begin{array}{c}
h_{x}{ }^{\prime}-h_{x}=f^{\prime}\left[\tan ^{-1}\left[\tan \left(\theta_{x}\right) \cos (\alpha)\right]-\theta_{x}\right] \\
h_{y}{ }^{\prime}-h_{y}=0
\end{array}\right.
$$

it can be seen that for the line field of view $\theta_{x}=0$, the image wander is zero at different angles $\theta_{y}$. For the condition of $\theta_{x} \neq 0$ and $\alpha \neq 0$, the image wander always exists during scanning.

The existence of projection distortion is because the optical axis of the imaging system is changed by the angle $\alpha$, but $\theta_{x}$ is still based on the original optical axis. Figure 3 shows a schematic diagram of the new projection relationship. Where $\mathrm{Oz}$ is the original optical axis. When the optical axis is in the state $\mathrm{B}, \theta_{y}=\alpha$, the new optical axis changes to $\mathrm{Oz}^{\prime}$. The projection point of $\mathrm{OI}_{\mathrm{y}}$ on the original optical axis $\mathrm{Oz}$ is $C$. The intersection of $\mathrm{I}_{\mathrm{y}} C$ and the new optical axis is $C^{\prime} . \mathrm{I}_{\mathrm{x}}{ }^{\prime} C^{\prime}$ is parallel to $\mathrm{I}_{\mathrm{x}} C$.

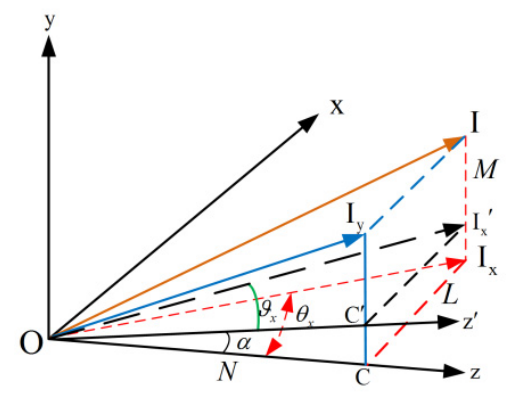

Figure 3. A schematic diagram of the new projection relationship. Oz is the original optical axis. When the optical axis is in the state $\mathrm{B}, \theta_{y}=\alpha$, the new optical axis changes to $\mathrm{Oz}$. The projection point of $\mathrm{OI}_{\mathrm{y}}$ on the original optical axis $\mathrm{Oz}$ is $C$. The intersection of $\mathrm{I}_{\mathrm{y}} \mathrm{C}$ and the new optical axis is $C^{\prime}$. $\mathrm{I}_{\mathrm{x}}{ }^{\prime} C^{\prime}$ is parallel to $\mathrm{I}_{x} C$. The angle between $\mathrm{OI}_{x}{ }^{\prime}$ and the new optical axis is $\vartheta_{x}$, which can be defined the field angle in the $x$ direction.

The angle between $\mathrm{OI}_{x}{ }^{\prime}$ and the new optical axis $\mathrm{Oz}^{\prime}$ is $\vartheta_{x}$, and we define $\vartheta_{x}$ as the field angle in the $\mathrm{x}$ direction. $L, M, N$ are the direction cosines of $\mathrm{OI}$ in the original optical axis coordinate system.

$$
\tan \left(\theta_{x}\right)=\frac{L}{N^{\prime}}, \cos (\alpha)=\frac{N}{\mathrm{OC}^{\prime}}, \tan \left(\vartheta_{x}\right)=\frac{L}{\mathrm{OC}^{\prime}}
$$

Using Equation (9), we can get the Equation (10):

$$
\tan \left(\vartheta_{x}\right)=\tan \left(\theta_{x}\right) \cos (\alpha)
$$

It is assumed that the relationship among the image height, the focal length of the imager and the incident angle of the imaging system in Figure 1 is shown in Equation (11):

$$
\left\{\begin{array}{l}
h_{x}=f^{\prime} \vartheta_{x} \\
h_{y}=f^{\prime} \theta_{y}
\end{array}\right.
$$

Considering that $\mathrm{y}$-direction of the FOV is the scanning direction. When the change angle of the optical axis is $\alpha$ and the mirror rotates $\alpha / 2$, the image point coordinates $\left(h_{x}{ }^{\prime}, h_{y}{ }^{\prime}\right)$ of the chief ray OI on the FPA will come to be expressed by Equation (12):

$$
\left\{\begin{array}{c}
h_{x}{ }^{\prime}=f^{\prime} \tan ^{-1}\left[\tan \left(\theta_{x}\right) \cos (\alpha)\right]=f^{\prime} \vartheta_{x} \\
h_{y}{ }^{\prime}=f^{\prime} \theta_{y}
\end{array}\right.
$$


Calculating the difference between image points $\left(h_{x}{ }^{\prime}, h_{y}{ }^{\prime}\right)$ and $\left(h_{x}, h_{y}\right)$ in Equation (13),

$$
\left\{\begin{array}{l}
h_{x}{ }^{\prime}-h_{x}=0 \\
h_{y}{ }^{\prime}-h_{y}=0
\end{array}\right.
$$

it can be seen that the image wander caused by the projection distortion during the scanning of the mirror is zero.

When the FSM is put in the middle of the optical system, an imaging system is schematically shown in Figure 4, which includes a front-end afocal telescope, a FSM, an imager and an FPA. Back-scanning with a FSM behind the afocal telescope provides an agile method to increase the integration time for the FPA by compensating for movement of the imaging system. As the system scans in object space, the FSM tilts to attempt to keep the image fixed on the FPA during the integration time. In Figure 4, the imager's FOV is scanned through the afocal telescope's FOV. The green box represents the imager's FOV at the earlier point in State A. The blue box represents the imager's FOV at the later point in State B. The black circle represents the afocal telescope's FOV. The gray arrow represents the scanning direction. The corresponding back-scanned imager's FOV in object space are also showed. The afocal telescope's FOV is scanning to the down, while the FSM moves to keep the imager's FOV (the orange box) fixed during the integration time.
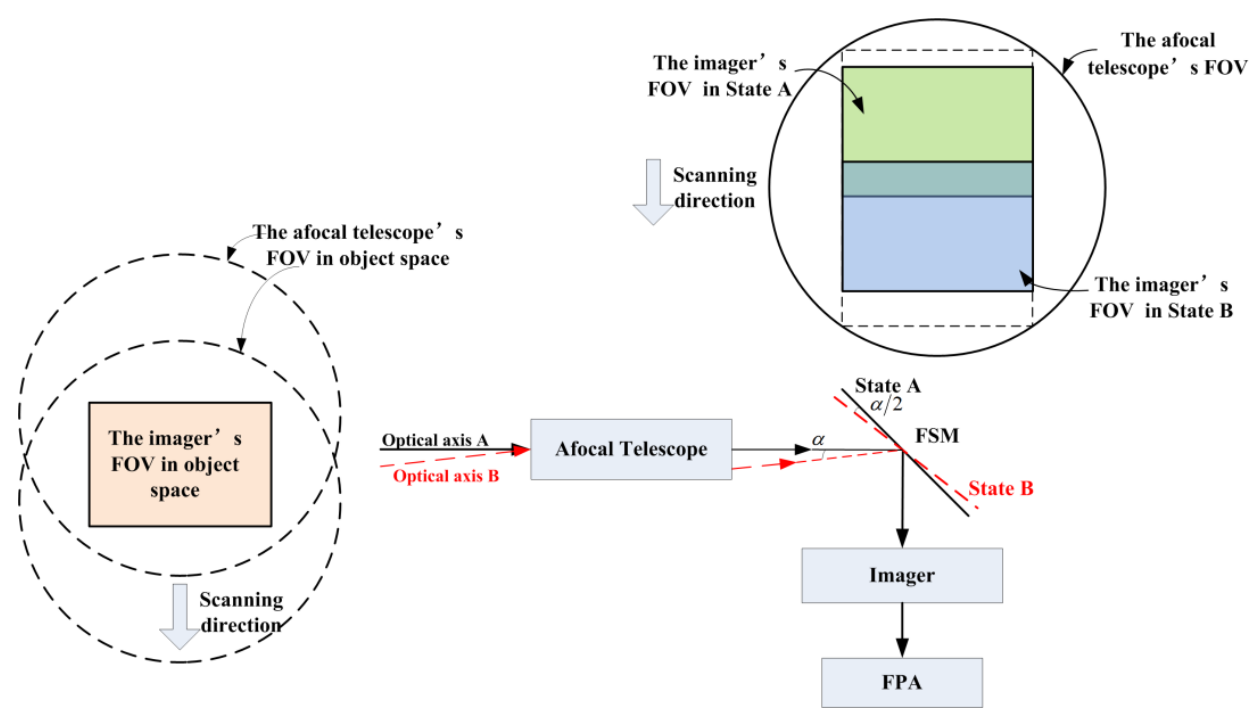

Figure 4. Schematic diagram of an imaging system. The imaging system includes a front-end afocal telescope, a fast steering mirror (FSM), an imager and a focal plane array (FPA).

Assuming that the angular magnification of the afocal telescope is $A_{\text {mag }}$ and the focal length of the imager is $f^{\prime}{ }^{\prime}$, in order to meet Equation (14), the following conditions should be satisfied:

$$
\left\{\begin{array}{l}
h_{x}=A_{\text {mag }} f_{\mathrm{c}} \vartheta_{x}=f_{\mathrm{c}}\left(A_{\mathrm{mag}} \vartheta_{x}\right) \\
h_{y}=A_{\mathrm{mag}} f_{\mathrm{c}}^{\prime} \theta_{y}=f_{\mathrm{c}}\left(A_{\mathrm{mag}} \theta_{y}\right)
\end{array}\right.
$$

At this time, the relationship of the imager can satisfy $h=f^{\prime} \theta$, but the afocal telescope needs to satisfy the following relationship in Equation (15):

$$
\left\{\begin{array}{l}
\vartheta_{x}{ }^{\prime}=A_{\operatorname{mag}} \vartheta_{x} \\
\theta_{y^{\prime}}=A_{\operatorname{mag}} \theta_{y}
\end{array}\right.
$$


Modifying Equation (11), we can get the Equation (16):

$$
\left\{\begin{array}{c}
h_{x}=f^{\prime} \tan \left[\frac{\tan \left(\theta_{x}\right)}{\sqrt{1+\tan ^{2}\left(\theta_{y}\right)}}\right] \\
h_{y}=f^{\prime} \theta_{y}
\end{array}\right.
$$

The power series expansion to $h_{x}$ can be obtained in Equation (17):

$$
h_{x} \sim f^{\prime} \theta_{x}+\left(-\frac{f^{\prime} \theta_{x}}{2}+\frac{f^{\prime} \theta_{x}^{3}}{3}\right) \theta_{y}^{2}+\frac{f^{\prime} \theta_{x}}{24} \theta_{y}^{4}+\ldots
$$

Unlike the conventional field mapping of equation $h=f^{\prime} \tan \theta$ and $h=f^{\prime} \theta$, the optimal field mapping according to Equations $h_{x}=f^{\prime} \vartheta_{x}, h_{y}=f^{\prime} \theta_{y}$ are not rotationally symmetric. It has an anamorphic nature. This non-rotationally symmetric field mapping deletes all field points wander during back-scanning.

\section{Optical System Design}

The mathematical model of non-rotationally symmetric field mapping between object space and image space is established in Section 2. In this section, we want to design optical system which can implement these field mappings.

The design specifications are shown in Table 1. The spectral band is 3.7-4.8 $\mu \mathrm{m}$ (medium-wave infrared). The focal length of the optical system is $500 \mathrm{~mm}$. The F-number of the optical system is 4 . The detector is a two-dimensional FPA having $1280 \times 1024$ pixels. The pixel pitch of the FPA is $15 \mu \mathrm{m}$. The magnification of the afocal telescope is $5 \times$. The FOV of the frame is $2.2 \times 1.76$ degrees. The scanning speed of optical system is $40 \mathrm{deg} / \mathrm{s}$ and the normal integration time is $10 \mathrm{~ms}$. Consequently, the back-scan is over \pm 0.4 degrees in object space, and then the FOV of the afocal telescope is $2.2 \times 2.56$ degrees.

Table 1. Design specifications.

\begin{tabular}{cc}
\hline Characteristics & Performance \\
\hline Spectrum & $3.7-4.8 \mu \mathrm{m}$ \\
Focal length & $500 \mathrm{~mm}$ \\
F/\# & 4 \\
FPA array format & $1280 \times 1024$ \\
Pixel pitch & $15 \mu \mathrm{m}$ \\
Magnification of the afocal telescope & $5 \times$ \\
Scanning speed of optical system & $40 \mathrm{deg} / \mathrm{s}$ \\
Normal integration time & $10 \mathrm{~ms}$ \\
FOV of the afocal telescope & $2.2 \times 2.56 \mathrm{degrees}$ \\
FOV of the frame & $2.2 \times 1.76 \mathrm{degrees}$ \\
\hline
\end{tabular}

It should be noted that the main purpose of this manuscript is to explain the image points wander during back-scanning. In order to simplify the design, the imager is set as an ideal lens, and the practical problems of infrared optical system design such as secondary imaging and cold stop matching are not considered.

The optical system designed according to the field mapping condition $h_{x}=f^{\prime} \vartheta_{x}, h_{y}=f^{\prime} \theta_{y}$ is shown in Figure 5. The afocal telescope is consisted of six lenses. From left to right, the lens 1 , the lens 3 , the lens 4 , and the lens 5 are made of silicon and have a positive power. The lens 2 and the lens 6 are made of germanium and have a negative power. The entrance pupil is placed on the front surface of the lens 1 . The rear surface of the lens 2 is a quadratic aspherical surface. The lenses 3 and 4 are field lenses, and have a small power. The rear surface of the lens 3 and the front surface of the lens 4 are freeform surfaces. The remaining surfaces are spherical. The FSM is placed on the exit pupil of the 
afocal telescope, and the optical axis is turned by 90 degrees. The ideal lens imager focuses the scene onto the FPA.

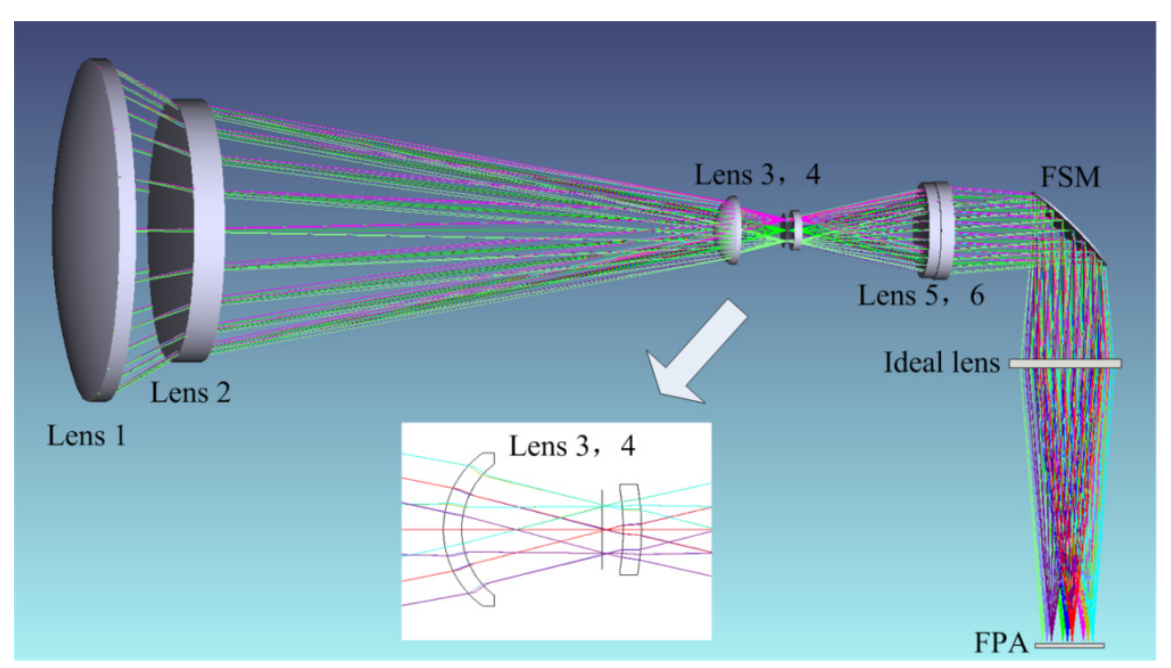

Figure 5. Optical system according to the field mapping conditions $h_{x}=f^{\prime} \vartheta_{x}, h_{y}=f^{\prime} \theta_{y}$

The freeform surfaces used in this example are extended XY polynomial. The sagittal deviation $\Delta z$ between the freeform surface and the standard spherical surface is expressed by Equation (18):

$$
\Delta z=a_{1} x^{2}+a_{2} y^{2}+a_{3} x^{4}+a_{4} x^{2} y^{2}+a_{5} y^{4}+a_{6} x^{6}+a_{7} x^{4} y^{2}+a_{8} x^{2} y^{4}+a_{9} y^{6}
$$

The above non-rotationally symmetric freeform surfaces are used to control the tilt of the chief ray in the $X$ and $Y$ directions, which can produce an anamorphic aberration to meet the Equation (11).

A special macro is coding to constrain the relationship among the image height, the focal length and the incident angle of the imaging system to meet Equation (11). At the same time, a multi-configuration is designed to include five different angles of the FSM. In the process of optimization, the image heights of the same FOV are limited to be equal. If this new field mapping is strictly restricted, the image quality of the system will be degraded, and the image distortion will be very serious. For the actual optical systems design, it is necessary to balance aberrations to meet the special specifications, such as the maximum allowable distortion when the FSM does not move, the maximum image wander during back-scanning and the minimum MTF under the Nyquist frequency. For the above optical system, the distortion weight should be magnified as much as possible while the MTF is close to the diffraction limit. For this optical system, the maximum distortion is less than $0.46 \%$ within the FPA. Figure 6 shows the grid distortion map. In order to visualize the distortion characteristics, the distortion deviation is enlarged by 20 times. As can be seen from Figure 6, the distortion is similar to pincushion distortion, but the distortion is not rotationally symmetric. The deviation of the actual image with the theoretical field point in $x$ direction is larger than that in $y$ direction.

During the exposure of the FPA, the back-scan is over \pm 0.4 deg in object space. The curves in Figure 7 indicate motion (in units of pixel IFOV) of the chief ray at nine image points during the back-scan operation. As we can see in Figure 7, except for the central image point, the other off-axis image points have image wander. The image wander at the four edge fields of view is the largest. Compared with the case when the fast steering mirror has no motion, the maximum image blur during the back-scan is only 0.22 pixels. The image point motion caused by projection distortion is well controlled. 


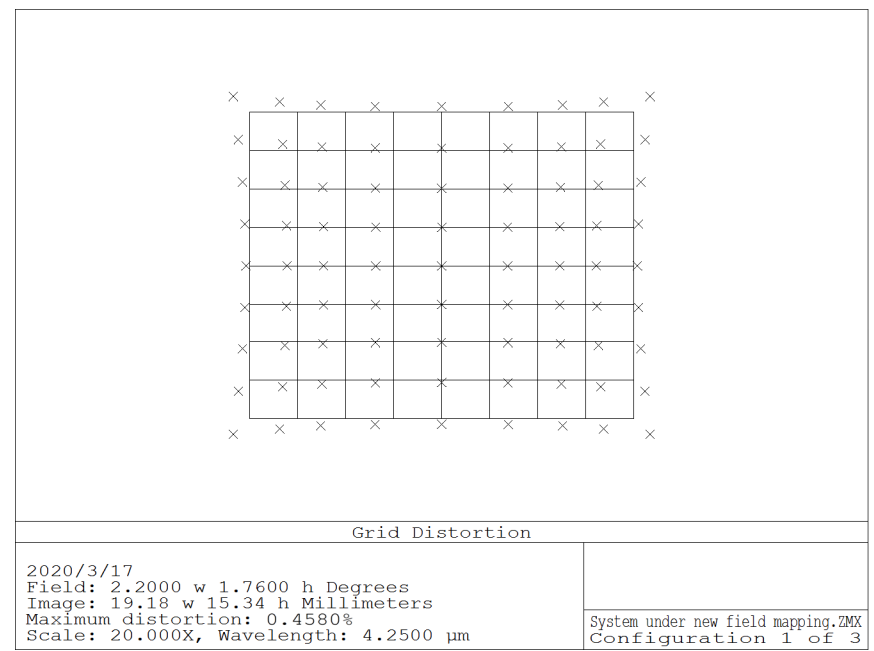

Figure 6. Grid distortion. The X point is the actual field point, and the grid is the theoretical field point. In order to visually display the distortion characteristics, the distortion deviation is enlarged by 20 times.

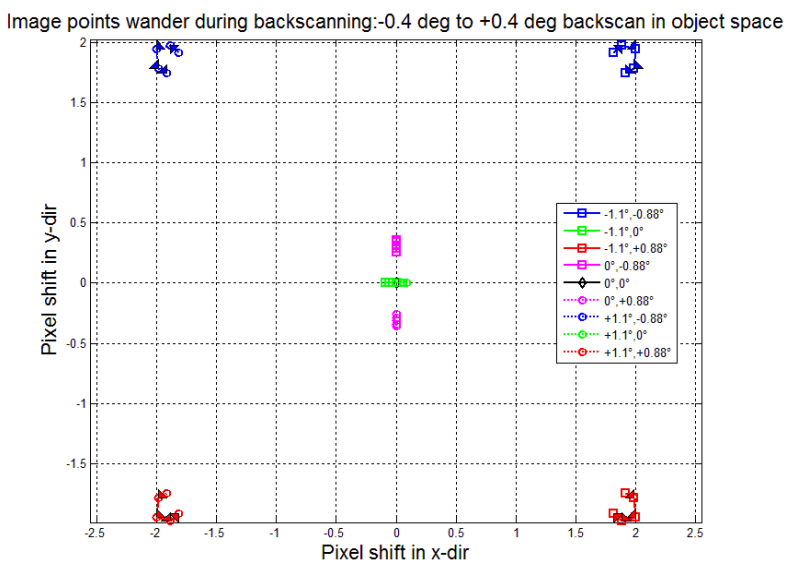

Figure 7. Image points wander during back-scanning under field mapping condition $h_{x}=f^{\prime} \vartheta_{x}, h_{y}=f^{\prime} \theta_{y}:-0.4$ deg to +0.4 deg back-scan in object space.

\section{Comparison and Analysis}

In order to explain the distortion characteristic differences of optical systems under different field mapping equations during back-scanning, another two medium-wave infrared optical systems are successfully designed in meeting the same design specifications in Table 1.

The optical system designed by Zemax software according to the field mapping condition $h=f^{\prime} \tan \theta$ is shown in Figure 8. The afocal telescope is composed of five lenses. From left to right, the lens 1 , the lens 3 , and the lens 4 are made of silicon and have a positive power. The lens 2 and the lens 5 are made of germanium and have a negative power. The entrance pupil is placed on the front surface of the lens 1 . The rear surface of the lens 2 is a secondary aspheric surface, and the remaining surfaces are spherical. The lens 3 is a field lens, and the optical power is very small. The fast steering mirror is placed on the exit pupil of the afocal telescope, and the optical axis is turned by 90 degrees. The imager focuses the scene onto the FPA. The optical system have good imaging performance and low pupil aberrations. 


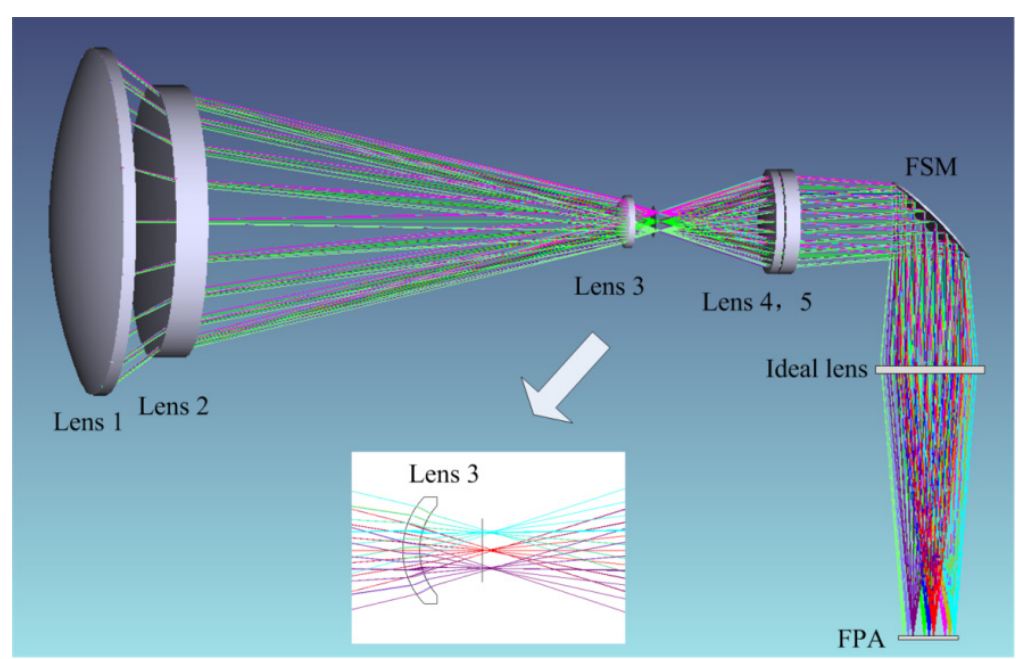

Figure 8. Optical system according to the field mapping condition $h=f^{\prime} \tan \theta$

The optimization commands of Zemax software are utilized to set the relationship among the image height, the focal length and the incident angle of the imaging system to meet Equation (2). For the above system, the MTF is close to the diffraction limit. The maximum distortion is less than $0.02 \%$ within the FPA. When FSM remain still, the distortion of the optical system is controlled well. Figure 9 shows the grid distortion map. In order to visualize the distortion characteristics, the distortion deviation is enlarged by 200 times. As can be seen from the figure, this optical system displays symmetrical barrel distortion. In this moment, the distortion seemed to be controlled and the image quality seemed to be well. Further, the FSM placed behind the afocal telescope could be considered to have nothing to do with the image quality by optical designers. Actually, the movement required to track an image viewed through a rotating mirror is a nonlinear function of the mirror movement, but this fact is always neglected [20].

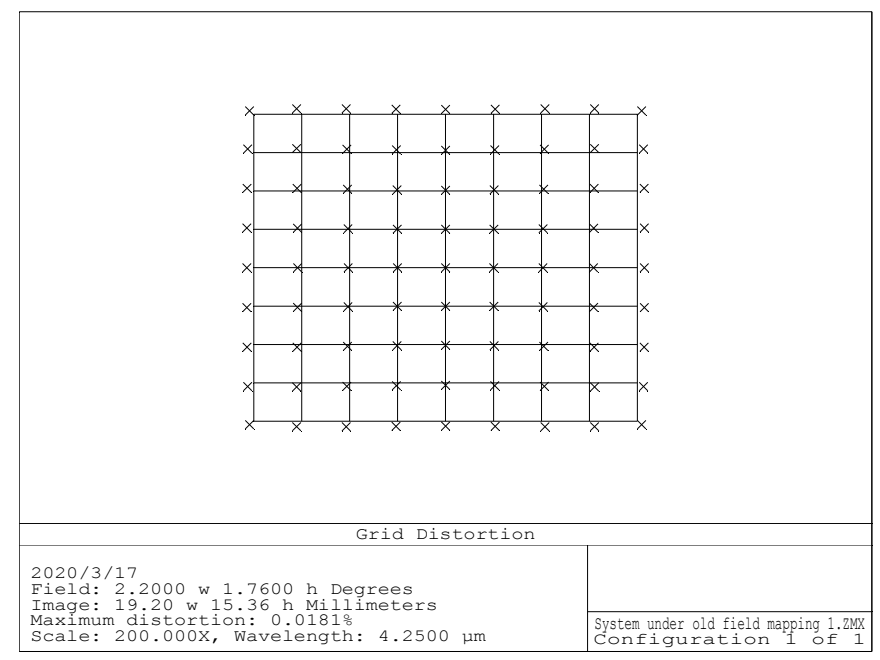

Figure 9. Grid distortion of the optical system in Figure 8. The X point is the actual field point, and the grid is the theoretical field point. In order to visually display the distortion characteristics, the distortion deviation is enlarged by 200 times.

During the exposure of the FPA, the back-scan is over \pm 0.4 deg in object space. The lines in Figure 10 indicate motion (in units of pixel IFOV) of the chief ray at nine image points during the back-scan operation. As can be seen from the figure, except for the central image point, the other off-axis image points have image wander. The image wander at the four edge fields of view is the 
largest. Compared with the case when the fast mirror has no motion, the maximum distortion image shift is 3.13 pixels. This image wander will severely degrade the image quality.

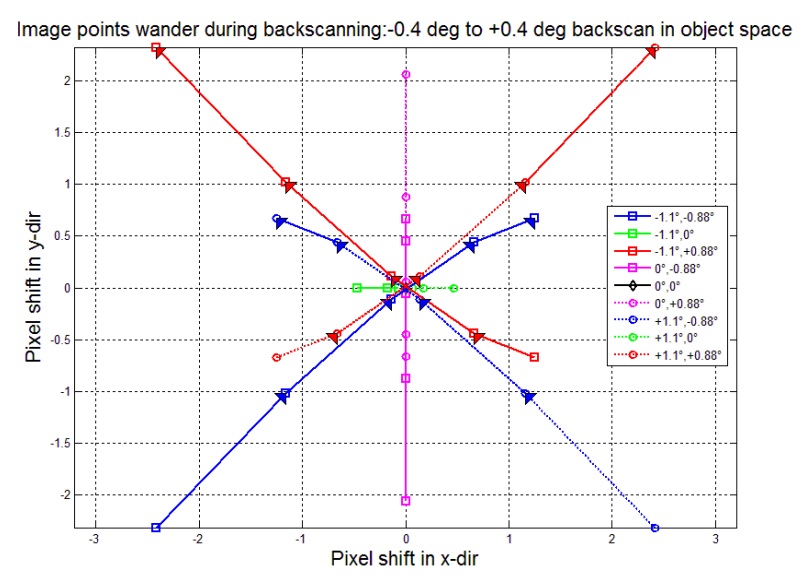

Figure 10. Image points wander during backscanning under field mapping condition $h=f^{\prime} \tan \theta$ : -0.4 deg to +0.4 deg back-scan in object space.

The optical system in Figure 8 is optimized according to the condition $h=f^{\prime} \theta$. Only the lens radius, air distance, and aspheric coefficient are slightly different, and the rest remain the same. The optimization commands of Zemax software are utilized to set the relationship among the image height, the focal length and the incident angle of the imaging system to meet Equation (6). For the above system, the MTF is close to the diffraction limit. For this optical system, the maximum distortion is less than $0.012 \%$ within the FPA. When fast steering mirror does not move, the distortion of the optical system is controlled well. Figure 11 shows the grid distortion map. In order to visualize the distortion characteristics, the distortion deviation is enlarged by 200 times. As can be seen from the figure, this optical system still displays symmetrical barrel distortion. The field mapping of Equation (6) minimizes image wander for field points along a single axis. Line-scan systems can use this mapping and achieve adequate results. However, this optimal one-dimensional field mapping is not ideal for two-dimensional imaging systems.

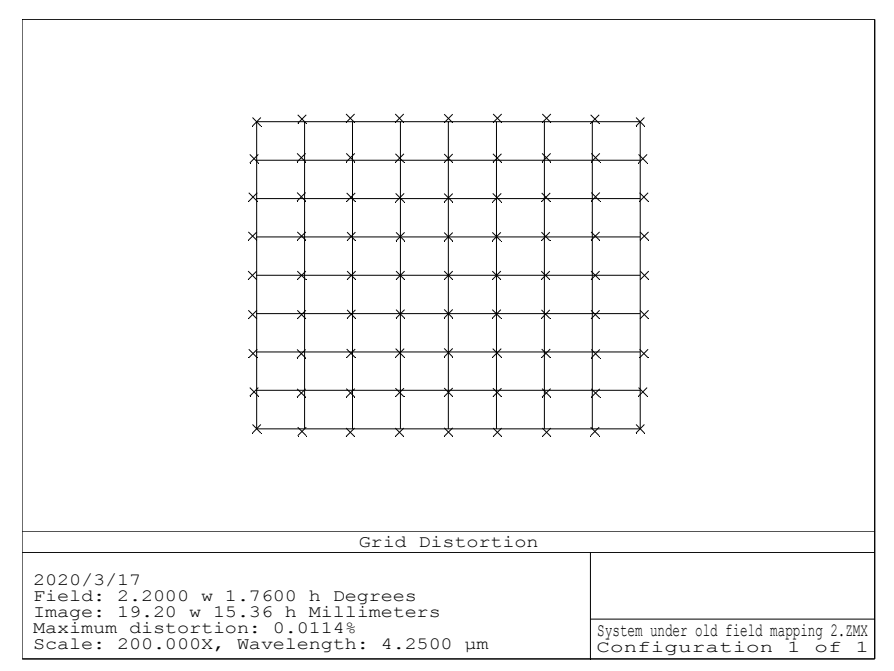

Figure 11. Grid distortion. The $X$ point is the actual field point, and the grid is the theoretical field point. In order to visually display the distortion characteristics, the distortion deviation is enlarged by 200 times.

During the exposure of the FPA, the back-scan is over \pm 0.4 deg in object space. The lines in Figure 12 indicate motion (in units of pixel IFOV) of the chief ray at nine image points during the 
back-scan operation. As can be seen from the figure, except for the central image point, the other off-axis image points have image wander. The image wander at the four edge fields of view is the largest. Compared with the case when the fast mirror has no motion, the maximum distortion image shift is 3.18 pixels. The image wander is similar to that in Figure 12. This image wander will also severely degrade the image quality.

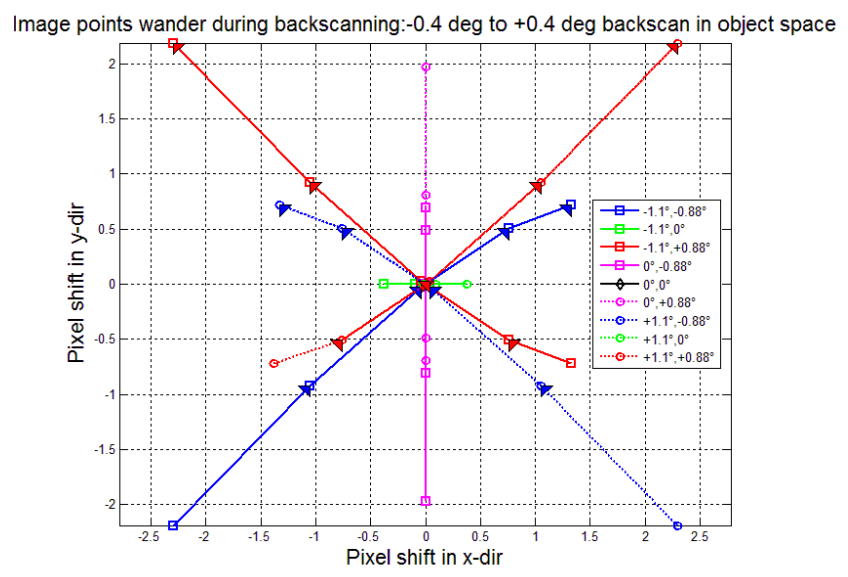

Figure 12. Image points wander during back-scanning under field mapping condition $h=f^{\prime} \theta:-0.4$ deg to +0.4 deg back-scan in object space.

Additionally, we compared the optical system based on the proposed model $h_{x}=f^{\prime} \vartheta_{x}, h_{y}=f^{\prime} \theta_{y}$ to the optical systems based on the model $h=f^{\prime} \tan \theta$ and $h=f^{\prime} \theta$ in our experiments, and the distortion and image wander are selected as the evaluation parameters. Table 2 lists the maximum distortion when the FSM does not move and the maximum image point wander during back-scanning in the previous three optical systems. The optical systems that meet $h=f^{\prime} \tan \theta$ and $h=f^{\prime} \theta$ have a distortion of less than $0.02 \%$, which is approximately no distortion. At this time, the movement of the image points are caused by the projection distortion during the movement of the FSM. For the optical system that meets the conditions of non-rotational projection, the maximum distortion is less than $0.46 \%$. When the FSM does not move, a large distortion remains in the optical system, which is more than 23 times that of the conventional optical systems. During the rotation of the FSM, the maximum wander of the image points on the FPA is only 0.22 pixels, and the remaining distortion just compensates for the projection distortion, which verifies the effectiveness of the proposed method, as "one cannot make an omelet without breaking eggs". However, in order to achieve no image point wander during the back-scanning, the optical system is more complicated than the traditional optical systems, and non-rotationally symmetric free-form surfaces are required to achieve a non-rotational symmetry field mapping.

Table 2. Distortion characteristics and image wander.

\begin{tabular}{ccc}
\hline Characteristics & $\begin{array}{c}\text { Maximum Distortion When FSM } \\
\text { Does Not Move }\end{array}$ & $\begin{array}{c}\text { Maximum Image Wander during } \\
\text { Back-Scanning }\end{array}$ \\
\hline Optical system under $h=f^{\prime} \tan \theta$ & $<0.02 \%$ & 3.13 pixels \\
Optical system under $h=f^{\prime} \theta$ & $<0.012 \%$ & 3.18 pixels \\
Optical system under & $<0.46 \%$ & 0.22 pixels \\
$h_{x}=f^{\prime} \vartheta_{x}, h_{y}=f^{\prime} \theta_{y}$ & & \\
\hline
\end{tabular}

\section{Conclusions and Future Work}

Addressing the problems of off-axis field point wander and image blurring caused by the use of FSM in back-scanned step/stare imaging system, this manuscript proposes a non-rotationally symmetric field mapping method for back-scanned step/stare imaging system, which can make all field points 
stable on the FPA during back-scanning. The mathematical model of non-rotationally symmetric field mapping between object space and image space is established. Meanwhile, a back-scanned step/stare imaging system based on the model is designed, in which this non-rotationally symmetric mapping can be implemented with an afocal telescope including freeform lenses. Experimental results show that this non-rotationally symmetric field mapping can provide the highest contrast images for back-scanned step/stare imaging system.

The future work includes three aspects. Firstly, the actual optical imaging systems will be utilized to verify the impact of image wander on SNR through quantitative imaging experiments. Secondly, due to the fact that the application of the freeform surfaces will always increase the development cycle and funding of the imaging systems, to meet this non-rotationally symmetric field mapping, we will try to introduce a little tilt and eccentricity on the field lens of the afocal telescope or the certain lens of the imager to avoid the application of freeform surfaces. Finally, the differences between the theoretical image wanders and the actual image wanders for different FOVs will be researched when the system has the manufacturing errors, the assembly errors, the position errors and the control errors of the FSM.

Author Contributions: Conceptualization, Q.F. and X.Z.; methodology, Q.F.; software, Q.F.; validation, J.Z. and G.S.; writing—original draft preparation, Q.F.; writing—review and editing, Q.F., S.Z. and M.L. All authors have read and agreed to the published version of the manuscript.

Funding: This work was supported by National Natural Science Foundation of China [grant number 61505201].

Acknowledgments: The authors would like to thank the anonymous reviewers for their useful comments and critical remarks which helped to improve this paper.

Conflicts of Interest: The authors declare no conflict of interest. We have purchased the genuine software license of Zemax and stated the company name in our article. The funders had no role in the design of the study.

\section{References}

1. Sun, C.; Ding, Y.; Wang, D.; Tian, D. Backscanning step and stare imaging system with high frame rate and wide coverage. Appl. Opt. 2015, 54, 4960-4965. [CrossRef] [PubMed]

2. Venkateswarlu, R.; Er, M.H.; Tam, S.C.; Chan, C.W.; Choo, L.C. Design considerations of IRST system. Proc. SPIE 1997, 3061, 591-602.

3. Nouguès, P.O.; Baize, P.; Roland, F.; Olivier, J.F.; Renaudat, M. Third-generation naval IRST using the step-and-stare architecture. Proc. SPIE 2008, 6940, 69401B.

4. de Ceglie, S.U.; Moro, M.L.; Vita, R.; Neri, A.; Barani, G.; Cavallini, M.; Quaranta, C.; Colombi, G. SASS: A bi-spectral panoramic IRST-Results from measurement campaigns with the Italian Navy. Proc. SPIE 2010, 7660, 766007.

5. Barani, G.; Olivieri, M.; Luison, C.; Rossi, A.; Diani, M.; Acito, N. Development of a panoramic third generation IRST: Initial study and experimental work. Proc. SPIE 2013, 8704, 87040.

6. Fortunato, L.; Colombi, G.; Ondini, A.; Quaranta, C.; Giunti, C.; Sozzi, B.; Balzarotti, G. SKYWARD: The next generation airborne infrared search and track. Proc. SPIE 2016, 9819, 98190K.

7. Olson, C.; Theisen, M.; Pace, T.; Halford, C.; Driggers, R. Model development and system performance optimization for staring infrared search and track (IRST) sensors. Proc. SPIE 2016, 9820, $98200 \mathrm{~B}$.

8. Cho, M.; Jun, Y.; Dribusch, C.; Ryu, J.; Poczulp, G.; Liang, M.; Lee, S.; Han, J.Y.; Jeong, U.; Kim, S.; et al. Design of the fast steering secondary mirror assembly for the Giant Magellan Telescope. Proc. SPIE 2018, 10706, 1070607.

9. Hilkert, J.M.; Kanga, G.; Kinnear, K. Line-of-sight kinematics and corrections for fast-steering mirrors used in precision pointing and tracking systems. Proc. SPIE 2014, 9076, 90760F.

10. Netzer, Yishay, Line-of-sight steering and stabilization. Opt. Eng. 1982, 21, 210196. [CrossRef]

11. Baumann, J.L.; Dixon, M.D. Precision Stabilization Requirements For Long-Range Acquisition Systems. Proc. SPIE 1979, 187, 90-97.

12. Germann, L.; Gupta, A. Inertial Line-of-Sight Stabilization Using Fine Steering Mirrors. In Proceedings of the Conference on American Astronautically Society Guidance and Control Conference, Minneapolis, MN, USA, 15-17 August 1988. 
13. Stewart, J.B.; Bifano, T.G.; Cornelissen, S.; Bierden, P.; Levine, B.M.; Cook, T. Design and development of a 331-segment tip-tilt-piston mirror array for space-based adaptive optics. Sens. Actuators A 2007, 138, 230-238. [CrossRef]

14. Tapos, F.; Edinger, D.J.; Hilby, T.R.; Ni, M.S.; Holmes, B.C.; Stubbs, D.M. High bandwidth fast steering mirror. Proc. SPIE 2005, 5877, 60-73.

15. Gutierrez, H.L.; Gaines, J.D.; Newman, M.R. Line-of-sight stabilization and back scanning using a fast steering mirror and blended rate sensors. In Infotech@Aerospace 2011; American Institute of Aeronautics and Astronautics: Reston, VA, USA, 2011; pp. 1-8.

16. Miller, J.L.; Way, S.; Ellison, B. Design challenges regarding high-definition electro-optic/infrared stabilized imaging systems. Opt. Eng. 2013, 52, 061310. [CrossRef]

17. Petrushevsky, V.; Freiman, D.; Diamant, I.; Giladi, S.; Leibovich, M. Common aperture multispectral spotter camera: Spectro XR. Proc. SPIE 2017, 10433, 043315.

18. Driggers, R.G.; Halford, C.E.; Theisen, M.J.; Gaudiosi, D.M.; Olson, S.C.; Tener, G.D. Tener, Staring array infrared search and track performance with dither and stare step. Opt. Eng. 2018, 57, 053101. [CrossRef]

19. Wang, L.; Liu, X.; Wang, C. Modeling and design of fast steering mirror in image motion compensation for backscanning step and stare imaging systems. Opt. Eng. 2019, 58, 103105. [CrossRef]

20. Goodson, J. Dynamics of an Image Viewed through a Rotating Mirror. J. Opt. Soc. of Am. 1979, 69, 771-775. [CrossRef]

21. Royalty, J. Development of Kinematics for Gimballed Mirror Systems. Proc. SPIE 1990, 1304, $262-274$.

22. Gershon, G.; Avnon, E.; Brumer, M.; Freiman, W.; Karni, Y.; Niderman, T.; Ofer, O.; Rosenstock, T.; Seref, D.; Shiloah, N.; et al. $10 \mu \mathrm{m}$ pitch family of InSb and XBn detectors for MWIR imaging. Proc. SPIE 2017, 10177, $101771 \mathrm{I}$.

23. Sharifi, H.; Roebuck, M.; Terterian, S.; Jenkins, J.; Tu, B.; Strong, W.; De Lyon, T.J.; Rajavel, R.D.; Caulfield, J.; Nosho, B.Z.; et al. Advances in III-V bulk and superlattice-based high operating temperature MWIR detector technology. Proc. SPIE 2017, 177, 101770U.

24. Dhar, N.K.; Dat, R.; Sood, A.K. Advances in infrared detector array technology. In Optoelectronics-Advanced Materials and Devices; Pyshkin, S.L., Ballato, J.M., Eds.; InTech Open: Rijeka, Croatia, 2013; Chapter 7.

(C) 2020 by the authors. Licensee MDPI, Basel, Switzerland. This article is an open access article distributed under the terms and conditions of the Creative Commons Attribution (CC BY) license (http://creativecommons.org/licenses/by/4.0/). 